\title{
Outlook for $b$ Physics at the LHC in ATLAS and CMS
}

\author{
Attila Krasznahorkay Jr. ${ }^{1,2}$ for the ATLAS and CMS collaborations \\ 1- University of Debrecen - Dept. of Experimental Physics \\ 4010, Debrecen, Egyetem tér 1. - Hungary \\ 2- CERN - PH Dept. \\ CH-1211, Geneva 23 - Switzerland
}

\begin{abstract}
An overview is presented for the planned B-physics programme of the ATLAS and CMS experiments at the LHC. The physics programmes of both experiments have been prepared for the different running conditions of the accelerator. Analyses and their expected sensitivities are presented, which are planned for different luminosity configurations of the LHC.
\end{abstract}

\section{Introduction}

ATLAS [2] and CMS [3] are general purpose particle detectors that will run at the Large Hadron Collider (LHC) at CERN. Both of them provide good tracking, calorimetry and muon detection. They were designed mainly for high- $\mathrm{p}_{\mathrm{T}}$, discovery physics. B physics events on the other hand contain mostly low- $\mathrm{p}_{\mathrm{T}}$ particles, which means that for these measurements the detectors usually have to be used in modes they were not optimised for.

The study of B-physics presents a good opportunity to check the Standard Model predictions at a high perturbative order, search for new physics, constrain the CKM matrix elements and to provide new information on long-distance QCD effects in matrix elements of the tensor currents.

Proton-proton collisions will happen at $\sqrt{s}=14 \mathrm{TeV}$ at the LHC which results in a $\sigma(b \bar{b})=500 \mu \mathrm{b}$ cross section for $b \bar{b}$ pair production. This means that at low luminosity LHC running $\left(\mathrm{L}=2 \times 10^{33} \mathrm{~cm}^{-2} \mathrm{~s}^{-1}\right) b \bar{b}$ pairs will be produced with a rate of about $10^{6} \mathrm{~Hz}$. Since only on the order of about $10 \mathrm{~Hz}$ can be written to storage for B-physics in both experiments, trigger systems with high rejection powers are needed to select the events of interest.

\section{B hadron property measurements}

Despite the noisy environment of the LHC, the huge B hadron production statistics will allow precise measurements of their properties. Current studies show that the first precision measurements will be possible with about $10 \mathrm{fb}^{-1}$ of data, which could be gathered with one year of data taking at low luminosity.

\subsection{Inclusive $b$ cross section}

Today the shape of the transverse momentum and angular distributions as well as the azimuthal angular correlations of $\mathrm{B}$ flavoured hadrons are reasonably well described by perturbative QCD. However the observed cross-sections at the Tevatron, HERA and LEP are larger than most ${ }^{\mathrm{a}} \mathrm{QCD}$ predictions. Three mechanisms contribute to the beauty production in hadron colliders: gluon-gluon fusion and $q \bar{q}$ annihilation; flavour excitation and gluon

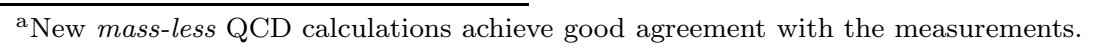


splitting. It is important to measure the B-hadron $\mathrm{p}_{\mathrm{T}}$ spectra within large range to be able to disentangle the contributions of these mechanisms.

CMS studied the measurement of the in-

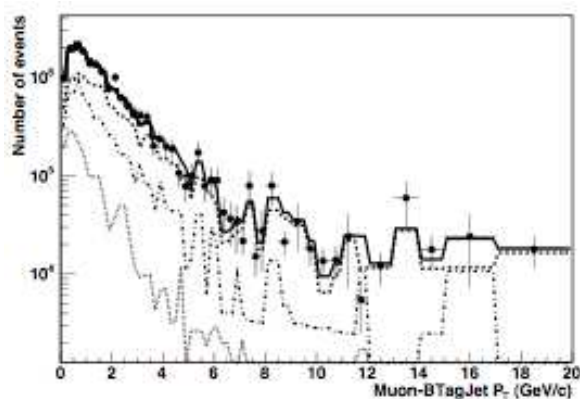
clusive $b$ cross section in the channel with a $b$-tagged jet and a muon in the final state [4]. The transverse momentum of the muon with respect to the $b$-jet axis can be used to effectively discriminate $b$ events from the background, as is demonstrated in Figure 1. Using a maximum likelihood fit, the cross section can be extracted from this distribution.

With an integrated luminosity of $10 \mathrm{fb}^{-1}$ CMS expects to study the $b$ production cross section on a sample of 16 million $b$

Figure 1: Fit of the muon $\mathrm{p}_{\mathrm{T}}$ spectrum with respect to the closest $b$-tagged jet. The contributions of tagged muons from $b$ events (dashed curve), $c$ events (dot-dashed curve) and light quark events (dotted curve) as defined by the fit are shown.

events. The current estimate shows that the measurement can reach $1.5 \mathrm{TeV} / \mathrm{c}$ as the highest measured transverse momentum for B hadrons.

\section{$2.2 B_{c}$ meson property measure- ments}

A feasibility study was done in the CMS experiment to measure the mass and lifetime of the $B_{c}$ meson [5]. They concluded that with the first $\mathrm{fb}^{-1}$ of data CMS will be able to reconstruct roughly $120 B_{c}^{+} \rightarrow J / \psi \pi^{+}$with $J / \psi \rightarrow \mu^{+} \mu^{-}$events.

A Gaussian fit of the mass distribution of these events results in a mass resolution of 22.0 (stat.) \pm 14.9 (syst.) $\mathrm{MeV} / \mathrm{c}^{2}$. The corresponding lifetime uncertainty is $0.044($ stat. $) \pm$ 0.010 (syst.) ps.

\section{CP violation studies}

The full proper-time and angular analyses of B events allow the investigation of several parameters of physics interest. If the Standard Model expectations are correct, the weak phase $\left(\phi_{s}\right)$ will not be measured with a useful significance, but a deviation from the Standard Model could be detected. Studying the decay of heavy flavoured hadrons also presents the opportunity to measure some of the CKM matrix elements in a direct way.

\subsection{Measurement of $\sin (2 \beta)$}

ATLAS's sensitivity to measure $\sin (2 \beta)$ in the $B_{d}^{0} \rightarrow J / \psi K_{s}$ decay was estimated on fully simulated Monte Carlo samples with a maximum likelihood method. With dedicated triggers available for the $J / \psi \rightarrow \mu^{+} \mu^{-}\left(e^{+} e^{-}\right)$final states, ATLAS should achieve high sensitivity in this channel. The $J / \psi \rightarrow e^{+} e^{-}$channel will mainly be used to cross-check the results from $J / \psi \rightarrow \mu^{+} \mu^{-}$, and to slightly improve on them.

It is possible to tag the flavour of the $B_{d}^{0}$ mesons from tagging the flavour of the $b$ quark on the opposite side either with the jet charge or lepton flavour of that decay. Combining 
all the tags, a precision of 0.01 on $\sin (2 \beta)$ could be achieved with $30 \mathrm{fb}^{-1}$ of data at low luminosity. The systematic uncertainty on the measurement is estimated to be 0.005 .

\section{$3.2 \Delta m_{s}$ measurement}

In $B_{s}^{0} \rightarrow D_{s} \pi, B_{s}^{0} \rightarrow D_{s} a_{1}$ the probability to detect an initially pure $B_{s}^{0}$ as $B_{s}^{0}\left(p_{+}\right)$or as $\bar{B}_{s}^{0}\left(p_{-}\right)$is:

$$
p_{ \pm}(t)=e^{-\Gamma t}\left(\cosh \frac{\Delta \Gamma_{s}}{2} t \pm \cos \Delta m_{s} t\right) \frac{\Gamma^{2}-\Delta \Gamma_{s}^{2}}{2 \Gamma}
$$

From which the $\Delta m_{s}$ parameter can be derived. The projection of ATLAS's sensitivity to the $\Delta m_{s}$ parameter can be seen in Figure 2. As can be seen from the plot, a $5 \sigma$ limit could be obtained for CDF's recent measurement [6] already with $10 \mathrm{fb}^{-1}$ of data.

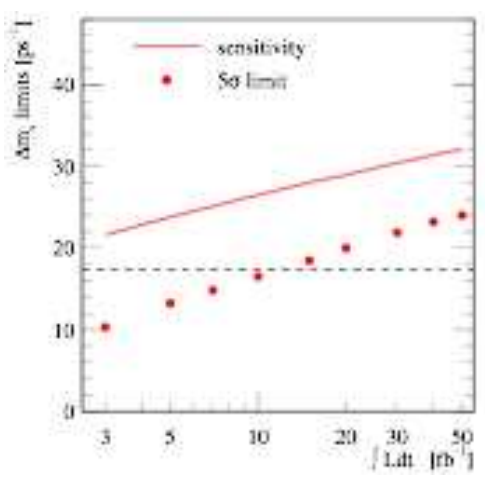

\section{$3.3 \phi_{s}$ measurement in $B_{s}^{0} \rightarrow J / \psi \phi$}

The $B_{s}^{0} \rightarrow J / \psi \phi$ decay leads to three final state helicity configurations. Their linear combinations are $\mathrm{CP}$ eigenstates with different $\mathrm{CP}$ parities. The experimental observables of the $B_{s}^{0} \rightarrow J / \psi \phi \rightarrow$

Figure 2: $\Delta m_{s}$ measurement limits as a function of the integrated luminosity. The dashed line represents the CDF measurement. $\mu^{+} \mu^{-} K^{+} K^{-}$decay are three independent angles and the $B_{s}^{0}$ proper time. They can be used in maximum likelihood fits to extract among other parameters the weak phase $\left(\phi_{s}\right)$.

ATLAS expects to be able to select about 270k signal events from $30 \mathrm{fb}^{-1}$ of data taken at low luminosity. This would allow to achieve a mass resolution on $B_{s}^{0}$ of $16.5 \mathrm{MeV}$ and a proper life-time resolution of $83 \mathrm{fs}$. The precision for determining $\phi_{s}$ from this sample $\left(\sigma\left(\phi_{s}\right)\right)$ is calculated to be 0.046 . The fit is also expected to provide $\Gamma_{s}$ and $\Delta \Gamma_{s}$ with $1 \%$ and $13 \%$ relative accuracy respectively.

In a similar analysis done by CMS [7] they found that the uncertainty on the weak phase determination with $10 \mathrm{fb}^{-1}$ of data is too large to make a measurement. It has to be noted though, that CMS's sensitivity will likely largely increase by introducing flavour tagging in their analysis.

\section{Rare B-decays}

Flavour changing neutral current decays $(b \rightarrow d, s)$ are forbidden at tree level in the Standard Model, and only occur at loop level. They are sensitive to the CKM matrix elements $\left|V_{t d}\right|$, $\left|V_{t s}\right|$ and also to new physics beyond the Standard Model.

Each of the studied channels have two muons in their final state. This makes it possible to trigger on the events even at high LHC luminosity $\left(\mathrm{L}=10^{34} \mathrm{~cm}^{-2} \mathrm{~s}^{-1}\right)$. Properly selecting dimuon signatures by the trigger and rejecting fake di-muon candidates is of high importance. A study is going on in ATLAS to evaluate the trigger's impact/bias on selecting rare B-decay 
events. Preliminary results show no bias from the LVL1 2MU6 trigger on a signal sample of $B_{d} \rightarrow K^{0 *} \mu(6) \mu(4)$ events.

\section{1 $\Lambda_{b} \rightarrow \Lambda \mu^{+} \mu^{-}$decays}

In this process the forward-backward asymmetry $\left(A_{F B}\right)$ is very sensitive to Supersymmetry. ATLAS's sensitivity to measure $A_{F B}$ in this decay was measured. With $30 \mathrm{fb}^{-1}$ of data 800 signal events are expected to be selected, analysing which should enable clear separation between the Standard Model and some of it's extensions.

\section{$4.2 \quad B_{s}^{0} \rightarrow \mu^{+} \mu^{-}$decays}

The branching ratio of this decay is sensitive to Standard Model extensions. ATLAS showed that with $30 \mathrm{fb}^{-1}$ of data it would be able to select about 21 signal- with an expected additional 60 background events. The expected event yields with $100 \mathrm{fb}^{-1}$ of data at high luminosity running are about 92 signal with 900 background events.

CMS showed [8] that with $10 \mathrm{fb}^{-1}$ of data they would be able to select $6.1 \pm 0.6$ signal events which would allow them to make a $\operatorname{BR}\left(B_{s}^{0} \rightarrow \mu^{+} \mu^{-}\right)<1.4 \times 10^{-8}$ limit with a $90 \%$ confidence level.

\section{Conclusion}

The LHC will provide it's experiments with unprecedented statistics of heavy flavour quark production. The main physics interest in ATLAS and CMS will be to make precision measurements on B properties made possible with the large event statistics, and do measurements that can detect or constrain physics beyond the Standard Model. The B-physics program in both experiments is prepared for all luminosities of the LHC, with some analyses possible even at the highest of the planned luminosities.

\section{References}

[1] Slides: http: //indico. cern. ch/contributionDisplay . py? contribId=240\&sessionId=5\&conf Id=9499

[2] ATLAS detector and physics performance, Technical Design Report, CERN, Geneva, 1999.

[3] Michael Della Negra, Achille Petrilli, Alain Harvé and Lorenzo Foà, CMS Physics Technical Design Report, CERN, Geneva, 2006.

[4] V. P. Andreev, D. B. Cline and S. Otwinowski, "Measurement of open beauty production at LHC with CMS," CERN-CMS-NOTE-2006-120;

[5] X. W. Meng, J. Q. Tao and G. M. Chen, "Feasibility to study the $B_{c}$ meson at CMS," CERN-CMSNOTE-2006-118;

[6] A. Abulencia et al. [CDF - Run II Collaboration], Phys. Rev. Lett. 97 (2006) 062003 [AIP Conf. Proc. 870 (2006) 116] [arXiv:hep-ex/0606027].

[7] V. Ciulli, N. Magini, L. Wilke, T. Speer, K. Prokofiev, S. Shulga and T. Ilicheva, "Study of the decay $B_{s}^{0} \rightarrow J / \psi \phi \rightarrow \mu^{+} \mu^{-} K^{+} K^{-}, " \quad$ CITATION = CERN-CMS-NOTE-2006-121;

[8] U. Langenegger, "Study of $B_{s} \rightarrow \mu^{+} \mu^{-}$in CMS," arXiv:hep-ex/0610039. 